\title{
Ray-Tracing Based Registration for HRCT Images of the Lungs
}

\author{
Sata Busayarat ${ }^{1}$ and Tatjana Zrimec $^{1,2}$ \\ ${ }^{1}$ School of Computer Science and Engineering \\ University of New South Wales, Sydney, NSW, 2052, Australia \\ $\{$ satab, tatjana\}@cse.unsw.edu.au \\ ${ }^{2}$ Centre for Health Informatics \\ University of New South Wales, Sydney, NSW, 2052, Australia
}

\begin{abstract}
Image registration is a fundamental problem in medical imaging. It is especially challenging in lung images compared, for example, with the brain. The challenges include large anatomical variations of human lung and a lack of fixed landmarks inside the lung. This paper presents a new method for lung HRCT image registration. It employs a landmark-based global transformation and a novel ray-tracing-based lung surface registration. The proposed surface registration method has two desirable properties: 1) it is fully reversible, and 2) it ensures that the registered lung will be inside the target lung. We evaluated the registration performance by applying it to lung regions mapping. Tested on 46 scans, the registered regions were $89 \%$ accurate compared with the ground-truth.
\end{abstract}

\section{Introduction}

The amount of imaging information produced by today's High-resolution CT (HRCT) scanners is beyond the ability of a radiologist to process in normal clinical practice. A computer aided diagnosis (CAD) system is then required to scan the large number of images and draw radiologist's attention on fewer but diagnostically useful images. Such system must solve the classical problems in medical image analysis - segmentation, feature extraction and registration - in a specific domain. This paper will focus on the registration problem in HRCT images of the lungs.

A large body of literature has been published on registration techniques for medical imaging applications. Sonka [1] provided a recent survey of the field. Most of the works are in brain imaging domain 23, where the computer aided systems have relatively been well established. There are significantly fewer works reported on automatic image registration for HRCT images of the lung 456. Lung HRCT image registration problem is challenging because lung varies, in terms of size, shape and position, from person to person more significantly than, for example, the brain. Detecting landmarks for registration is also challenging because there are relatively few landmarks in lung images and lung disease processes may alter the appearances of those landmarks. 
In this paper, an automatic registration for HRCT images of the lung is presented. It combines a conventional point-based registration with a novel surface registration that uses three-dimensional ray tracing to find the correspondence between points on images from two lungs. The method is developed to register a sparse scan (has gaps between slices) to a lung model constructed from a volumetric scan. The method is then applied to determining lung regions, clinically meaningful areas inside the lung.

\section{3D Lung Model}

The three-dimensional lung model used in this work is created from a volumetric HRCT scan of relatively normal lungs. It contains information about anatomical landmarks required for the registration. Lung surfaces are automatically detected using adaptive thresholding and active contour snakes [7. We modified the method so that it takes the third-dimension continuity into account. Single-point landmarks, namely carina, sternum, spinal cord and lung roots, are manually identified to ensure the correctness. The lung surface model and the landmarks are shown in Fig. 1]

To determine the lung regions, we used a technique proposed by [8]. Additional landmarks are required. Trachea is detected using knowledge-directed thresholding. Hilum, a wedge-shaped depression of the mediastinal surface of each lung, is detected using the curvature analysis of the mediastinal lung surface. The detected landmarks are then used to characterize eight different lung regions as shown in Fig. 2 .
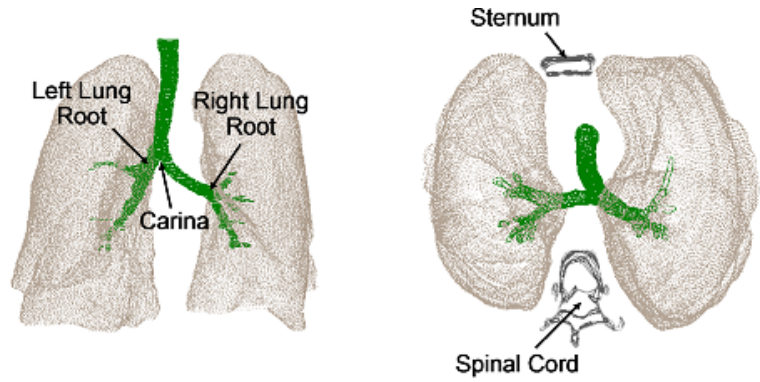

Fig. 1. A 3D lung model with landmarks

\section{Global Transformation Using Fixed Landmarks}

Apart from the significant anatomical variations in the human lung, different scanning protocols and different patient positions and orientations also contribute to the difficulties of comparing lungs from different HRCT scans. Global transformation or rigid image registration is used to align lungs for two different scans and help reduce the misplacing caused by the above factors. We use fixed 

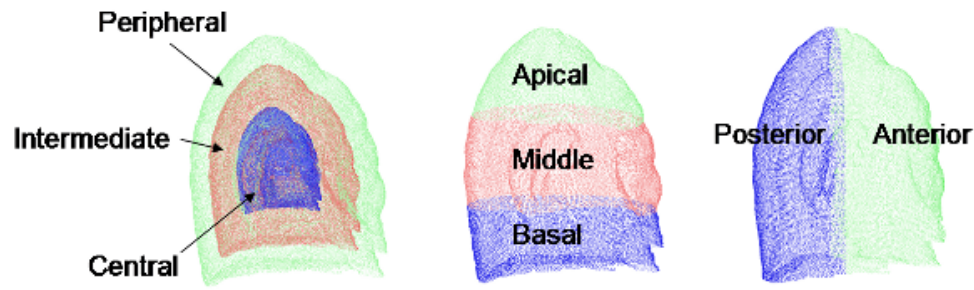

Fig. 2. Anatomical regions models. We use three models to represent eight different regions, including Perpheral-Intermediate-Central model (left), Apical-Middle-Basal model (middle) and Anterior-Posterior model (right).

reference points and three dimensional transformations for this registration. The reference points are stable anatomical landmarks, which are automatically detected. The transformations are performed in the following order: rotate, translate and scale. In our problem domain, registering a sparse scan to a 3D model, we choose to apply the transformation only to the sparse scan and keep the 3D model unchanged to save the computational time. The same operation will take approximately 20 times longer on volumetric data than on sparse data. However, after the registration, any results may have to be transformed back to the original data. Therefore, all the transformations we use are reversible.

\subsection{Rotation}

Rotation is used to eliminate the difference in patient orientation between two HRCT scans. We only rotate a scan in two dimensions, in the transverse plane, as we assume that there is no orientation difference in the longitudinal axis (patients always lay on horizontally flat beds). We use the image that contains the carina, the first bronchial bifurcation as the representative image to determine the suitable rotation. On the image, sternum and spinal cord are detected using knowledge-driven template matching 8]. Using the sternum and the spinal cord as landmarks (See Fig 1) the rotating angle is calculated so that, after the rotation, the lines between sternum and spinal cord's center points of two registered scans have the same orientations.

\subsection{Translation}

Translation is used to eliminate the difference in patient's lung position and scanning position. Lung root is used as the landmark for translation. Generally, the lung root is referred to as the hilum. Since we require a single-point landmark, not a region, we then defined the lung root as the point where the main bronchus enters the lung. Each lung of a sparse scan is translated so that its lung root is at the same position as the lung root of the $3 \mathrm{D}$ model.

\subsection{Scaling}

There are two main reasons why two lungs from two different scans have different sizes. One is obviously the anatomical variations. Factors like patient size, 
age, sex and race have contributions to the difference in lung size. The other reason is field-of-view setting of the scanner, which reflects the actual size (in millimeters) of a pixel. The field-of-view information is usually available in the image header. In this work, however, we only use imaging data as the input. The scaling is, therefore, done by using landmark-based transformation similar to the rotation and translation. The lung itself is used as the landmark. The dimensional difference of 3D bounding boxes of lungs in the sparse data and in the model is used to determine the required scaling. The results of the three transformations of a lung are shown in Fig. 3(a-d).

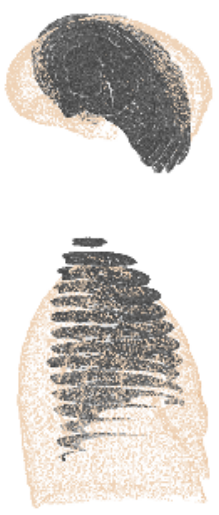

(a)
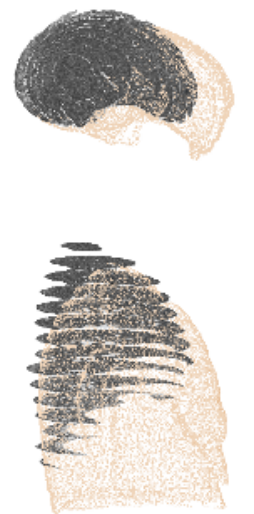

(b)
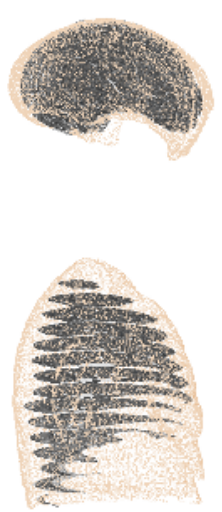

(c)
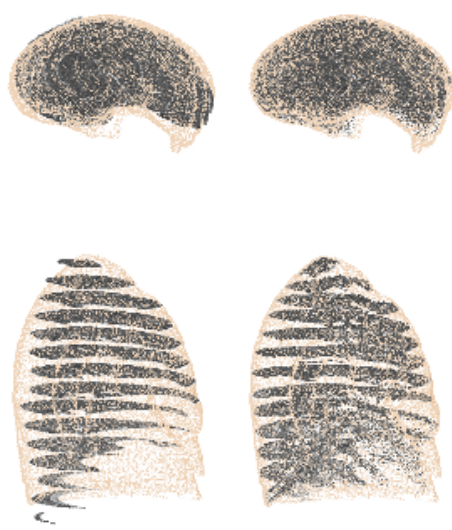

(d)

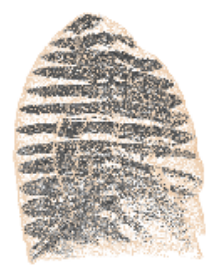

(e)

Fig. 3. Step-by-step results of lung registration in axial (upper row) and lateral (lower row) views. From left to right, (a) original lung from a sparse scan and the lung model, (b) result after rotation, (c) result after translation, (d) result after scaling and (e) final result after surface registration.

\section{Lung Surface Registration Using 3D Ray Tracing}

Ray tracing is a technique usually used in the field of computer graphics for real-time and offline rendering. In computer graphics, ray tracing works by projecting a ray of vision through each pixel on the screen from the observer 9 . Any objects in the scene that the ray hits, either directly or indirectly, will be used to determine the color of the on-screen pixel. We apply the same idea to 3D lung image registration, more specifically, registering a sparse scan to a $3 \mathrm{D}$ model. Instead of working out the colors of on-screen pixels, we use ray tracing to find correspondence between points on the surfaces of two lungs. In our case, the observer is the registration origin. The on-screen pixel corresponds to the point we want to register. The objects in the scene correspond to the surface of the lung.

Fig. 4(a) illustrates how the technique works. $S$ is a lung surface in a sparse scan of and $V$ is another lung surface in a volumetric scan used to build the lung model. $P$ is point in $S$ that we want to register to $V$. Firstly, we use the global transformation discussed in section 3 with $S$ to reduce the difference between $S$ 
and $V . P$ also has to be transformed since it is a point in $S$. The transformed $S$ and $P$ are called $S^{\prime}$ and $P^{\prime}$ respectively. We also have one registered point as the result of global transformation, which is the lung root. The lung root is used as the origin, $O$, for the ray tracing. We then draw a $3 \mathrm{D}$ ray from the origin through $P^{\prime}$. We trace the ray until it hits the lung surfaces of $S$ and $V$. The surface hitting points are called $P_{s^{\prime}}$ and $P_{v}$, respectively. The registered point, $R$, can then calculated using the distances from the origin to the surface hitting points as shown in Eq. 1 .

$$
R=O+\frac{\left|O P^{\prime}\right| \times\left|O P_{v}\right|}{\left|O P_{s^{\prime}}\right|}
$$

The equation suggests that the surface registration is reversible (we can work out $P^{\prime}$ given $R$ ). Combined with the reversible global transformation, a deformable model can be achieved at lower cost, as follows. We deform the sparse scan to fit the model and apply the reverse transformation to the model but only the part where the matching to the sparse scan exists. This generally saves about $90 \backslash \%$ of the computation required to deform a model because, a volumetric scan is approximately 20 times larger than a sparse scan. Fig. 4(c) shows a 2D cross section of the model deformed to fit a lung in Fig. 4(b).

The ray-tracing-based registration has another desirable property. Unlike other lung surface registration methods 465], it ensures that all the registered points are inside the lung model. A prove is given in Eq. 2. As a result, the deformed model is guaranteed to be a superset of the target sparse scan, which is suitable for internal lung structure mapping. Without this property, an inlung voxel of the sparse scan may be registered to a voxel in the volumetric scan that is outside the lung. Such voxel mapping is incorrect and further processing is required.

$$
\begin{aligned}
\max (R) & =O+\max \left(\left|O P^{\prime}\right| \times \frac{\left|O P_{v}\right|}{\left|O P_{s^{\prime}}\right|}\right) \\
& =O+\max \left(\left|O P^{\prime}\right|\right) \times \frac{\left|O P_{v}\right|}{\left|O P_{s^{\prime}}\right|} \\
& \left.=O+\left|O P_{s^{\prime}}\right| \times \frac{\left|O P_{v}\right|}{\left|O P_{s^{\prime}}\right|} \quad \text { (since } P^{\prime} \text { is in } S^{\prime}\right) \\
& =O+\left|O P_{v}\right|=P_{v}
\end{aligned}
$$

For the sparse scan, a completed lung surface needs to be approximated because a ray may hit the surface in between the slice gaps. The surface approximating algorithm works as follows. A number of triangle strips are generated by connecting lung boundary points between two consecutive slices. Scanline triangle filling algorithm [10] is then used to fill the triangle strips. The scanline algorithm is commonly used in computer graphics and is thus implemented in most of the VGA cards, which our surface approximation can benefit from.

The method is optimized for registering a large number of points from the same scan to the same model because, in most applications, every pixels inside 
the lung needs to be registered. Considering the fact that several points are registered using the same $3 \mathrm{D}$ ray and finding the surface hitting points $\left(P_{s}, P_{v}\right)$ is expensive, the surface hitting points are cached. An experiment has shown that the caching reduces the computation by about three times.

\section{$5 \quad$ Lung Region Mapping Using Lung Registration}

Lung registration has many applications. Berke et. al. [5] developed a lung surface registration technique and applied it to nodule registration problem. Zhang [4] used a deformable lung atlas to guide pulmonary fissure detection algorithm. In this work, we apply our registration technique to lung regions. We have built a model of eight lung regions. The model is deformed to fit the input scan using the reverse transformation. The region information from the model can then be directly transferred to the input scan. Fig. 4(d) shows an example of the registered peripheral, intermediate and central regions.

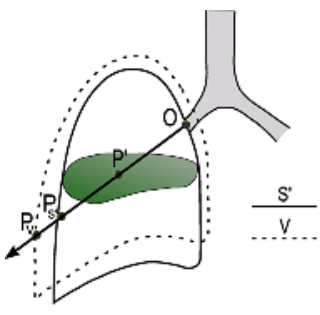

(a)

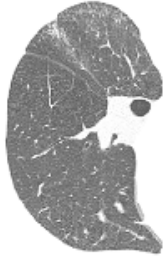

(b)

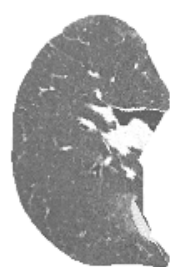

(c)

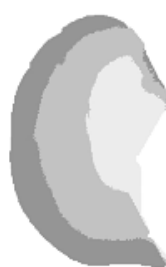

(d)

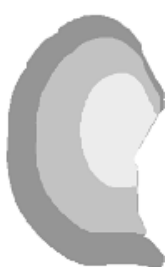

(e)

Fig. 4. Ray-tracing based lung registration method and results. From left to right, (a) illustrated ray-tracing technique, (b) input lung image, (c) deformed model that fits the input, (d) registered peripheral, intermediate and central regions, and (e) ground-truth of the regions.

\section{Evaluation}

HRCT scans from 46 patient studies were used for evaluation. The images were acquired as a part of normal clinical practice using standard imaging protocols. The images were taken using a SIEMENS scanner, with 512x512 spatial resolution, $1.0 \mathrm{~mm}$ slice thickness and $15 \mathrm{~mm}$ slice distance ( $\sim 20$ images per study). One volumetric HRCT scan, with $512 \times 512$ spatial resolution, $1.25 \mathrm{~mm}$ slice thickness and 0.8 slice distance (396 images), was used to construct the lung model.

We evaluated the performance of the proposed registration method by calculating the average surface-to-surface distance between a patient lung and the lung model, before and after the registration. The result in Table 1 indicates a slight distance reduction (20\%) after the global transformation and a significant reduction (78\%) after the surface registration. The differences between the left lungs are slightly greater than those of the right lungs. This may due to the heart artifact. 
Since another main goal of this work is to apply the registration method to map lung regions, we also evaluated the correctness of the registered regions. This reflects how well the method works with structures inside the lung, not only the lung surface. We used the result of an automatic region characterization algorithm proposed in [8] with manual corrections as the ground-truth to calculate the accuracy. An example of the ground-truth is shown in Fig. 4(e). The result in Table 2 shows that the region information in the lung model was successfully registered to the patient lungs, with $89 \%$ accuracy.

Table 1. Mean and startdard deviation of lung surface distance between the test lungs and the lung model, before and after the registration

\begin{tabular}{|c|c|c|c|}
\hline & \multicolumn{3}{|c|}{ Mean Distance Std. Dev. (pixels) } \\
\hline \hline & Before registration & After global transformation & After surface registration \\
\hline \hline Left lung & $26.2 \pm 5.7$ & $20.1 \pm 3.8$ & $6.1 \pm 0.9$ \\
\hline Right lung & $24.0 \pm 6.1$ & $19.3 \pm 4.4$ & $4.7 \pm 0.7$ \\
\hline Overall & $25.1 \pm 5.9$ & $19.7 \pm 4.1$ & $5.4 \pm 0.8$ \\
\hline
\end{tabular}

Table 2. Accuracy of the lung model's regions registered to the test lungs. The accuracy was calculated by comparing the registered regions with the ground truth.

\begin{tabular}{|c|c|}
\hline Regions & Accuracy (\%) \\
\hline \hline Peripheral-Intermediate-Central & 84 \\
\hline Apical-Middle-Basal & 87 \\
\hline Anterior-Posterior & 95 \\
\hline Overall & 89 \\
\hline
\end{tabular}

\section{Conclusions}

We have presented a new image registration method for HRCT images of the lung. We have adapted a ray tracing technique used in 3D graphics to lung image registration. It has two desirable properties: 1) the registration is reversible and 2) the registered lung is completely wrapped inside the lung model. They, together, make it possible create a deformable lung model using significantly less computation. The result showed that the proposed registration method reduces the lung surface difference between patient scans and the lung model by $78 \%$. We applied the method to the problem of registering lung regions, where the predefined region information was transferred from the model to a target lung. The registered regions were compared against the ground-truth $89 \%$ accuracy was achieved. This indicates that, even though we only use lung surface as the landmark for the registration, the internal lung volume was also registered accurately. However, a better result maybe obtained by using internal-lung landmarks, such as bronchial tree branching points, if one can solve the highly-altered-by-diseases problem of such landmarks. 


\section{Acknowledgement}

We would like to thank Dr. Peter Wilson for providing medical knowledge and guidance. This research was partially supported by the Australia Research Council through a Linkage grant (2002-2004), with I-Med Network Ltd. and Philips Medical Systems as partners.

\section{References}

1. Sonka, M., Fitzpatrick, J.: Handbook of Medical Imaging. Volume 2. SPIE Press (2000)

2. Ferrant, M., Nabavi, A., Macq, B., Jolesz, F., Kikinis, R., Warfield, S.: Registration of 3-D interoperative MR images of the brain using a finite-element biomechanical model. In: IEEE Trans. Med. Imag. Volume 20. (2001) 129-140

3. Kapouleas, I., Alavi, A., Alves, W., Gur, R., Weiss, D.: Registration of threedimensional MR and PET images of the human brain without markers. Radiology 181 (1991) 731-739

4. Zhang, L., Reinhardt, J.: 3D pulmonary CT image registration with a standard lung atlas. SPIE 3978 (2000) 67-77

5. Betke, M., Hong, H., Thomas, D., Prince, C., Ko, J.: Landmark detection in the chest and registration of lung surfaces with an application to nodule registration. Medical Image Analysis 7 (2003) 265-281

6. Betke, M., Hong, H., Ko, J.: Automatic 3D Registration of lung surfaces in computed tomography scans. In: MICCAI. (2001) 725-733

7. Papasoulis, J.: LMIK Anatomy and lung measurements using active contour snakes. Master's thesis, UNSW, Sydney, Australia (2003)

8. Zrimec, T., Busayarat, S.: A $3 \mathrm{~d}$ model of the human lung with lung regions characterization. In: ICIP. (2004) 1149-1152

9. Glassner, A.: An Introduction to Ray Tracing. Academic Press Limited (1989)

10. Foley, J.: Introduction to Computer Graphics. Addison-Wesley (1994) 